\title{
Performance Evaluation of Dynamic Computation Offloading Capability for Industrial Wearables
}

Citation

A. Ali, O. Galinina, J. Hosek and S. Andreev, "Performance Evaluation of Dynamic Computation Offloading Capability for Industrial Wearables," 2021 IEEE 32nd Annual International Symposium on Personal, Indoor and Mobile Radio Communications (PIMRC), 2021.

Version

Authors' camera-ready version

Link to publication

https://ieeexplore.ieee.org/document/9569484

Published in

2021 IEEE 32nd Annual International Symposium on Personal, Indoor and Mobile Radio Communications (PIMRC)

DOI

10.1109/PIMRC50174.2021.9569484

\section{License}

This publication is copyrighted. You may download, display, and print it for your own personal use. Commercial use is prohibited.

\section{Takedown policy}

If you believe that this document breaches copyright, please contact the authors, and we will investigate your claim.

\author{
BibTex entry \\ @INPROCEEDINGS\{ali20210Performance, \\ author $=\{$ Ali, Asad and Galinina, Olga and Hosek, Jiri and Andreev, Sergey $\}$, \\ booktitle $=\{2021$ IEEE 32nd Annual International Symposium on Personal, Indoor and Mobile Radio \\ Communications (PIMRC)\}, \\ title $=\{$ Performance Evaluation of Dynamic Computation Offloading Capability for Industrial Wearables $\}$, \\ year $=\{2021\}$, \\ do $=\{10.1109 /$ PIMRC50174.2021.9569484 $\}\}$
}




\title{
Performance Evaluation of Dynamic Computation Offloading Capability for Industrial Wearables
}

\author{
Asad $\mathrm{Ali}^{1,2}$, Olga Galinina ${ }^{1}$, Jiri Hosek ${ }^{2}$, and Sergey Andreev ${ }^{1}$ \\ ${ }^{1}$ Tampere University, Tampere, Finland \\ ${ }^{2}$ Brno University of Technology, Brno, Czech Republic \\ \{asad.ali, olga.galinina, sergey.andreev\}@tuni.fi, hosek@feec.vutbr.cz
}

\begin{abstract}
Extended Reality (XR) is a disruptive technology that will play an essential role in future society by creating an immersive human-machine interface. For their mass adoption, XR head-mounted devices have to be made light and sleek in design, which may require distributed computing capabilities, where high-end devices wirelessly offload computational tasks to the accompanying processing units. To satisfy demanding wireless connectivity requirements of the emerging XR applications, the devices are expected to rely on radio technologies that operate in millimeter-wave (mmWave) frequency range and require directional transmission. In this paper, we evaluate a dynamic system of distributed $3 D$ wearable networks operating in the mmWave band. We provide closed-form expressions for the session drop probability, the mean number of sessions that can run simultaneously, and its lower bound, which aid in understanding the impact of different parameters on the coexistence of dense directional wearable networks in the 3D space.
\end{abstract}

\section{INTRODUCTION}

The emerging concept of Industry 5.0 [1] extends Industry 4.0 by placing a stronger emphasis on cooperation between humans and machines. Creating seamless human-computer interfaces, Extended Reality (XR) can become one of the key enabling technologies to bridge the gap between the physical and digital worlds for professionals in industry [2].

Currently, XR technologies face multiple challenges associated with the form-factor and computation capabilities onboard the headsets, such as heat dissipation and weight requirements, low battery capacity, and computation constraints. One approach to overcome these limitations is to perform energy consuming and computationally expensive operations remotely. For example, XR headsets can be accompanied by a companion device, e.g., a puck or a smartphone [3], which performs computationally demanding tasks. These paired devices can be wirelessly tethered by a direct millimeter-wave (mmWave) link supported by, e.g., the IEEE 802.11ad/ay protocol or 5G NR sidelink as considered in 3GPP Release-17 [4] and beyond. The mmWave standards have the potential to enable data rates in the order of gigabits per second and, hence, meet the wireless connectivity requirements of XR applications.

A person equipped with an XR head-mounted display and an accompanying computation unit effectively carries a network of wearable devices, i.e., a wireless body-area network (WBAN). In close proximity, communicating devices of one owner may produce excessive interference and, hence, cause service interruptions at neighboring WBANs [5]. Since each WBAN operates independently, the degree of interference can potentially become prohibitive and degrade the quality of experience (QoE). For complex dynamic networks, systemlevel studies play a fundamental role in assessing the impact of interference subject to variable parameters and predicting the overall performance.

In research literature, system evaluation studies of mmWave WBANs have been conducted actively. In [6], the performance of mmWave WBANs is characterized with the help of stochastic geometry, and the proposed model describes the behavior of the system under different antenna gains. In [7], dense directional mmWave WBANs are analyzed in static settings, which, however, may not be representative of the dynamics exhibited by WBANs. In fact, distributed networks with directional communication, specifically in dense environments exhibit temporal dynamics which cannot be captured with the static models. In our previous works [8], [9], we had developed a framework for estimating the systemlevel performance of dynamic and independent WBANs while only considering the $2 \mathrm{D}$ setup.

In this paper, to provide more realistic estimates for the systems with 3D beamforming, we focus on a scenario featuring 3D directional mmWave WBANs, which is representative of, e.g., industrial setups.. We develop a model that can capture the impact of directional beams in 3D space and the randomness of transmission sessions. Furthermore, we derive closedform expressions for the session drop probability, the mean number of sessions that can run simultaneously, and its lower bound for the preset level of transmit power. The rest of this text is organized as follows. Section II introduces the system model and its underlying assumptions. Section III details the analytical framework, 3D modeling, and approximation proposed to obtain the metrics of interest. Finally, Section IV presents selected numerical results, and Section V concludes the paper.

\section{SySTEM MODEL}

In this section, we outline our assumptions adopted to abstract the considered scenario of independent WBANs.

\section{A. Deployment model}

We consider a finite-sized area of interest $S_{\mathrm{R}}$ where multiple WBANs operate concurrently. Each independent WBAN is 


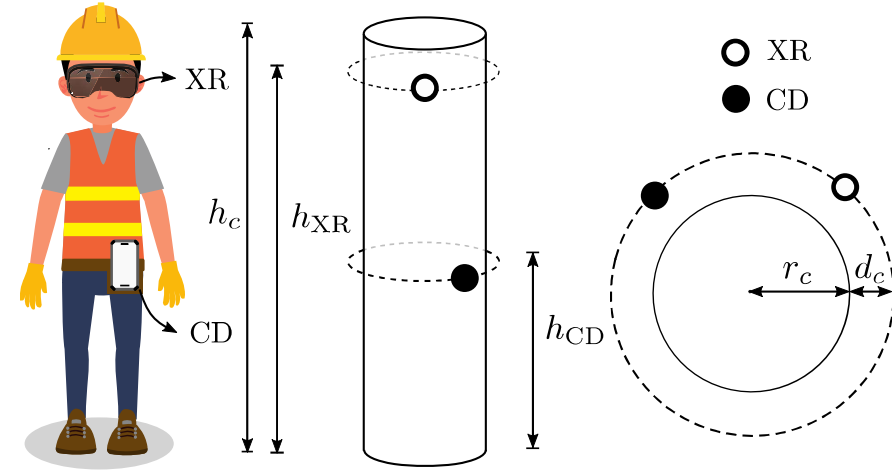

Fig. 1: Cylindrical model of human body.

associated with a user with two advanced wearable devices attached to the body, namely, an XR headset (termed XR) and its companion computation device (CD). All users are modeled as cylinders with radius $r_{c}$ and height $h_{c}$. The XR and the $\mathrm{CD}$ are located at heights $h_{\mathrm{XR}}$ and $h_{\mathrm{CD}}$, correspondingly. Both devices are placed randomly around the cylinder at a distance of $d_{c}$ from the cylinder surface, as shown in Fig. 1. The projections of the centers of users on the horizontal plane are distributed according to a Poisson Point Process (PPP). Using a simplified shape like a cylinder and constant heights enables tractable analysis and allows obtaining closedform expressions while maintaining the core system-level performance trade-offs.

\section{B. Session arrivals}

The XR transfers collected information (such as data from the camera, depth sensor, proximity sensor, IMUs, etc.) to the $\mathrm{CD}$, which then renders and streams back the data to be projected on the headset display. The period during which the $\mathrm{XR}$ and $\mathrm{CD}$ exchange the data is referred to as a session in the context of an XR application. We model the arrivals of users and the associated session requests with a Poisson process of intensity $\lambda$, and the session duration is exponentially distributed with mean $\mu^{-1}$.

We consider the session duration to be short so that the user and the devices remain stationary during transmission. We assume that the devices around the body do not change their locations while the session is ongoing. Hence, users may move between the times when the session terminates and a new session arrives. Based on this assumption, our model can be considered quasi-dynamic, i.e., the user moves between sessions, and during a session, the user remains static.

\section{Directionality model}

Both the XR headset and its companion computation device host mmWave transmitters allowing them to transfer data via a direct mmWave link. Following general principles of unlicensed mmWave protocols, we consider the data transmission during a session to be directional, whereas the reception to be omnidirectional. Hence, we model the receiving antenna gain as $G_{\mathrm{rx}}=1$ and the transmitting antenna gain as

$$
G_{\mathrm{tx}}=D_{0} \rho(\alpha),
$$

where $\alpha$ is the angular divergence from the antenna boresight of the transmit antenna beam that is perfectly aligned toward the receive antenna in $3 \mathrm{D}$ space, $D_{0}$ is the maximum directivity gain along the antenna boresight (i.e., $\alpha=0$ ), and $\rho(\alpha)$ is the gain reduction factor, which decreases the gain according to the angular divergence. The maximum directivity gain $D_{0}=\frac{2}{1-\cos \frac{\theta}{2}}$ is characterized by the half-power beamwidth $\theta$ of the transmit antenna array. Further, the gain reduction factor $\rho(\alpha)$ is defined as [10]

$$
\rho(\alpha)= \begin{cases}1-\frac{\alpha}{\theta}, & \alpha \leq \theta ; \\ 0, & \text { otherwise }\end{cases}
$$

The function $\rho(\alpha)$ is a piecewise function of the deviation angle $\alpha \in[0, \theta]$; the function scales as $\rho(\alpha) \in[0,1]$. The beam in 3D space has a pattern that is axially symmetrical around the axis of the antenna boresight. We assume perfect alignment between the devices communicating with each other during an active session. Here, perfect alignment could be a result of applying advanced beamforming techniques, such as those based on compressive sensing. We disregard the impact of side-lobes as they can be effectively suppressed by using appropriate windowing techniques.

\section{Intra-WBAN propagation}

The path loss is modeled according to $L(d)=C d^{-\kappa}$, where $\kappa$ is the propagation exponent, $C$ is the propagation constant, and $d$ is the distance between two devices. For any given transmit power $P_{\mathrm{tx}}$, the received power $P_{i}$ of the $i$-th user with an active session is given by

$$
P_{i}=\frac{P_{\mathrm{tx}} G_{\mathrm{rx}} G_{\mathrm{tx}}}{L(d)}=\frac{P_{\mathrm{tx}} D_{0}}{C d^{\kappa}}\left(1-\frac{\alpha}{\theta}\right) .
$$

Due to perfect alignment of beams between XR and CD, we can set $\alpha=0$, which translates to $\rho(\alpha)=1$. Under channel reciprocity, the received power $P_{i}$ at XR and CD devices of the $i$-th user can be readily defined as

$$
P_{i}=\frac{P_{\mathrm{tx}} D_{0}}{C d^{\kappa}} .
$$

Another important parameter is the receiver sensitivity threshold $P_{\mathrm{thr}}$ that denotes the minimum received power, for which the receiver can identify the desired signal. The maximum coverage distance along the antenna boresight for a given $P_{\mathrm{thr}}$ is, therefore, expressed by $R=\left(\frac{P_{\mathrm{tx}} D_{0}}{C P_{\mathrm{thr}}}\right)^{\frac{1}{\kappa}}$.

\section{E. Inter-WBAN interference and admission criteria}

The received power from neighboring devices is treated as interference. We introduce $I_{i \rightarrow n+1}$, which denotes the aggregated interference at the devices of the $(n+1)$-th user that originates from the devices of the $i$-th user, $i \leq n$. The term $I_{i \rightarrow n+1}$ is calculated as the maximum of the received 


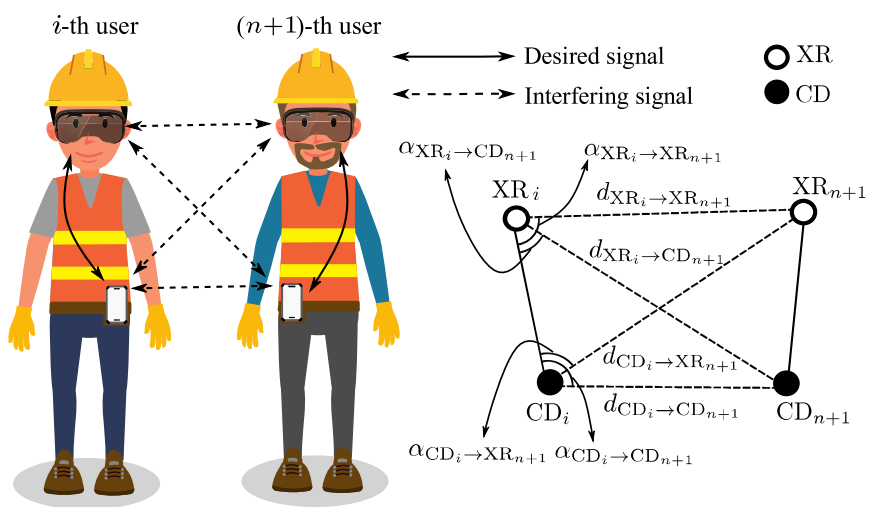

Fig. 2: Interference paths for two wearable networks.

powers along four paths, i.e., $\mathrm{XR}_{i} \rightarrow \mathrm{XR}_{n+1}, \mathrm{CD}_{i} \rightarrow \mathrm{XR}_{n+1}$, $\mathrm{XR}_{i} \rightarrow \mathrm{CD}_{n+1}$, and $\mathrm{CD}_{i} \rightarrow \mathrm{CD}_{n+1}$. Each path corresponds to different distances $d$ and angles of divergence $\alpha$ (illustrated in Fig. 2). The received interference that corresponds to a path $X_{i} \rightarrow Y_{n+1}$ is, thus, given as

$$
I_{X_{i} \rightarrow Y_{n+1}}= \begin{cases}\frac{P_{\mathrm{tx}} D_{0}}{C d_{X_{i} \rightarrow Y_{n+1}}^{\kappa}}\left(1-\frac{\alpha_{X_{i} \rightarrow Y_{n+1}}}{\theta}\right), & \alpha_{X_{i} \rightarrow Y_{n+1}} \leq \theta \\ 0, & \text { otherwise }\end{cases}
$$

where distance $d$ and angle of divergence $\alpha$ belong to the selected path $X_{i} \rightarrow Y_{n+1}$. Here, $X$ and $Y$ are either of the two devices, i.e., $\mathrm{XR}$ or $\mathrm{CD}$. We assume that a session can be initiated by the $(n+1)$-th user if and only if both devices do not receive any interference from the devices of any other users, that is, $I_{i \rightarrow n+1}$ is constrained by

$$
I_{i \rightarrow n+1}<P_{\mathrm{thr}} \forall i=1, \ldots, n .
$$

We note that (6) implies that the received power from the devices of the $i$-th user to the devices of the $(n+1)$-th user is lower than the receiver sensitivity for all four paths (see Fig. 2). As an example, we base our session admission criteria on the MAC procedure of the IEEE 802.11ad/ay protocol. Accordingly, the Distributed Coordination Function (DCF) controls medium access by using Request To Send (RTS) and Clear To Send (CTS) handshake before a data transmission to assess if the receiver is idle. A device senses the channel during Beacon Intervals (BI) and proceeds to transmission upon finding the channel idle.

In our model, the channel is considered idle if the interference power is under the receiver sensitivity $P_{\mathrm{thr}}$. If the channel is busy, a session cannot be initiated and will be dropped.

\section{PERFormance Evaluation Framework}

Due to Poisson arrivals and exponential service times, we can model the evolution of our system as a Markov process, which we denote as $S(t)$. At any time instant $t$, the state of $S(t)$ is defined by the number of active sessions within the area of interest and the 3D locations of the involved devices. We represent the position of XR and CD devices of the $i$-th user in $3 \mathrm{D}$ space by $\chi_{i}$ and the number of active sessions

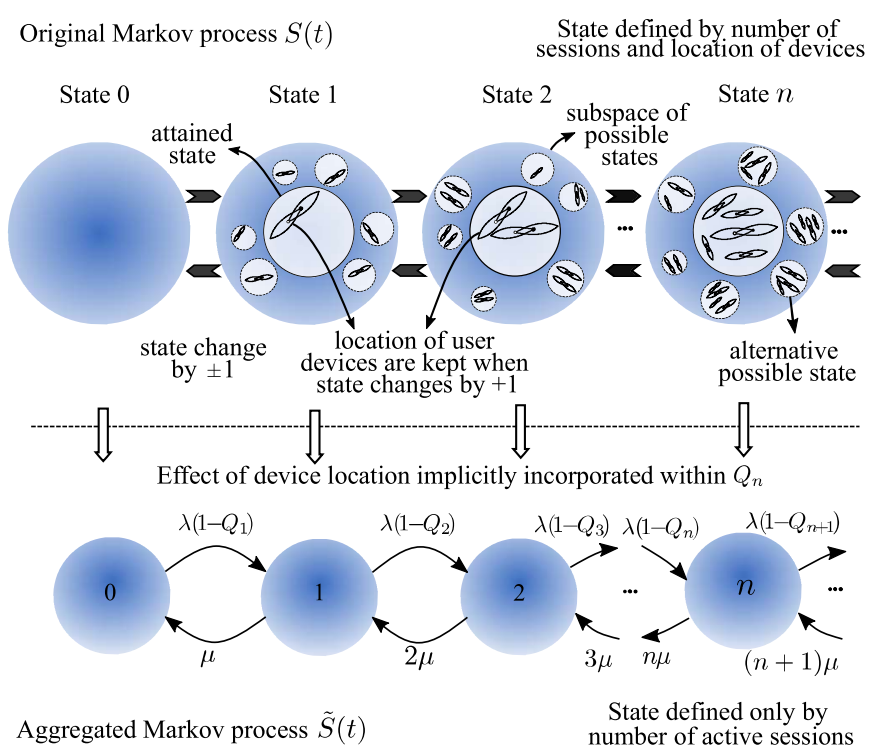

Fig. 3: Illustration of state aggregation from $S(t)$ to $\tilde{S}(t)$.

by $n$. Hence, a unique state of the Markov process $S(t)$ can be described as $\left[n \mid \chi_{1}, \chi_{2}, \ldots, \chi_{n}\right]$. For $n$ active sessions, the subset of states that have the same number of active sessions is infinite. For the process $S(t)$, the intensity of transitions from state $n$ back to state $n-1$ equals $n \mu$ as there are exactly $n$ active sessions and there is no dependency on the location. The transitions from state $n$ to state $n+1$ have the following intensities:

$$
\lambda \operatorname{Pr}\left\{I_{i \rightarrow n+1}<P_{\mathrm{thr}}, i=1, \ldots, n \mid\left[\chi_{1} \ldots, \chi_{n}\right]\right\},
$$

where the probability term corresponds to the event where the arriving pair passes the admission phase (6). Our admission policy requires to calculate distances $d$ and angles $\alpha$ from XR and CD devices for every user in the state $\left[n \mid \chi_{1}, \chi_{2}, \ldots, \chi_{n}\right]$. In addition, the number of states is uncountable, which makes the analysis cumbersome. Hence, we resort to a simplification of the process $S(t)$ attained by the state aggregation [11], [12].

\section{A. State aggregation for process $S(t)$}

Recall that for the process $S(t)$, the system is characterized by the number of active sessions $n$ and the locations of the corresponding devices. To eliminate the dependency on $\chi_{i}$, we combine all possible states in the subspace for $n$ active sessions into a single state $n$, regardless of the device locations as illustrated in Fig. 3.

A state of the resulting aggregated Markov process $\tilde{S}(t)$ is represented by the number of active sessions and may change only by \pm 1 . Particularly, the state changes either when a new session arrives given that the admission condition is satisfied, or when a session terminates after its service time in the system has elapsed. Most importantly, the next state of the system solely depends on its current state, i.e., the number of active pairs. To implicitly incorporate the memory of the process $S(t)$ into $\tilde{S}(t)$, we introduce drop probability $Q_{n+1}$. The term 
$Q_{n+1}$ is the probability of a new $(n+1)$-th session to be dropped when there are $n$ active sessions in the system. We illustrate the state aggregation procedure in Fig. 3.

Even though the locations are not considered explicitly, the system keeps a certain level of information on the previously admitted sessions through the introduced probability $Q_{n+1}$. Using $Q_{n+1}$, we move away from a complex system that keeps track of device locations to a memoryless process that only considers the number of active pairs.

\section{B. Stationary distribution of process $\tilde{S}(t)$}

The aggregated process reflects the main features of the original model so that it is feasible to derive the stationary distribution and other performance metrics. In this case, the transitions from state $n$ to state $n+1$ have the intensities $\lambda\left(1-Q_{n+1}\right)$ and the intensity of transitions from state $n$ to state $n-1$ is equal to $n \mu$. These simplifications render our simplified model to be a birth-death process.

After aggregation, the stationary state distribution of the resultant process $\tilde{S}(t)$ is given by:

$$
\pi_{m}=\pi_{0} \rho^{m} \frac{\prod_{n=1}^{m}\left(1-Q_{n+1}\right)}{m !}, m \geq 1,
$$

where $Q_{n+1}=\operatorname{Pr}\{(n+1)$-th session drop $\mid n$ active sessions $\}$, $\rho=\frac{\lambda}{\mu}$, and $\pi_{0}=\left(1+\sum_{m=1}^{\infty} \rho^{m} \prod_{n=1}^{m}\left(1-Q_{n+1}\right) / m !\right)^{-1}$.

With the steady-state distribution in (8), one can derive the average number of active sessions and the session drop probability as follows

$$
\begin{aligned}
\mathrm{E}[\mathrm{N}] & =\sum_{n=1}^{\infty} n \pi_{n}, \text { and } \\
\mathrm{P}_{\text {drop }} & =\pi_{0}+\sum_{n=1}^{\infty} Q_{n+1} \pi_{n} .
\end{aligned}
$$

Below, we describe the modeling of $Q_{n+1}$ for our system and the approximations designed to obtain closed-form solutions.

\section{Modeling session drop probability $Q_{n+1}$}

Our admission policy requires that interference at the devices arriving with new session requests should be less than the sensitivity threshold. During the neighbor's active session, a 3D beam originating from their device covers a volume in 3D space, within which the interference for the device with a new session request is higher than the sensitivity threshold. Hence, each active beam occupies a volume in 3D space, in which if a new device attempts to initiate a new session, the session will be dropped due to excessive interference.

In 3D space, $Q_{n+1}$ is equivalent to the probability of a new device emerging inside the volume of the $3 \mathrm{D}$ beam from any of the active devices. We reduce this problem from 3D space to 2D plane by considering the section of the beam on the 2D plane at the same height as the devices, as shown in Fig. 4. Given that the distance between XR and CD for the same user is short and that the beams are perfectly aligned, one can approximate the section of the beam on the $2 \mathrm{D}$ plane

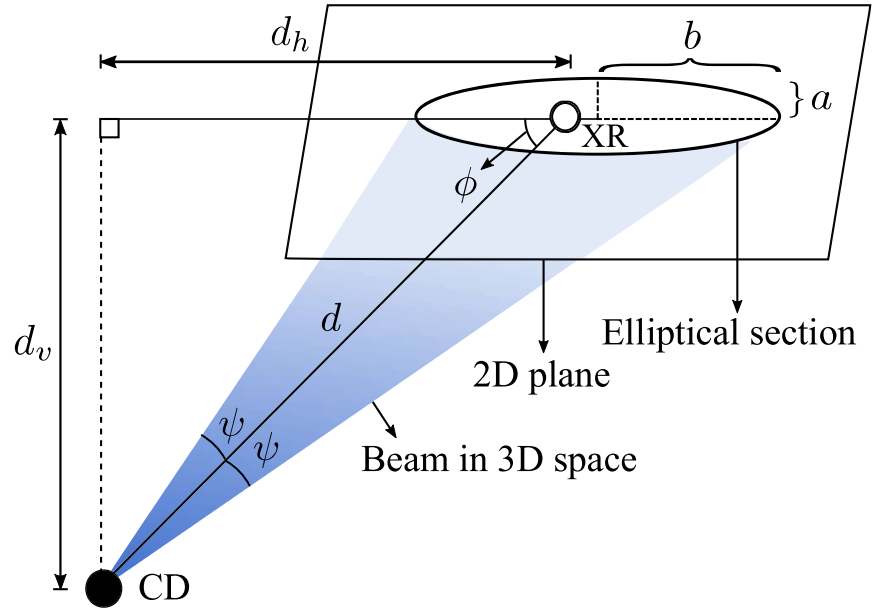

Fig. 4: Elliptical projection of 3D beam onto 2D plane.

with the section of a cone. With this approximation, we attain an elliptical section on the $2 \mathrm{D}$ plane, within which no device may appear due to excessive interference, i.e., $I_{i \rightarrow n+1}<P_{\mathrm{thr}}$. After our 2D simplification, the session drop probability can be obtained by using the mean area of the elliptical section in the $2 \mathrm{D}$ plane.

Lemma 1: We represent the expected elliptical area on 2D plane as $A_{\text {elip }}$, which is determined by semi-minor axis $a$ and semi-major axis $b$. We use expressions from [13] to obtain $a$ and $b$ as follows:

$$
a=\frac{d_{v} \sin (\psi)}{\sqrt{\sin ^{2}(\phi)-\sin ^{2} \psi}}, \quad b=\frac{d_{v} \sin (2 \psi)}{2\left(\sin ^{2}(\phi)-\sin ^{2} \psi\right)}
$$

where $\phi$ is the angle between the horizontal plane cutting the cone and the axis of the cone, $\psi$ is the semi-apex angle of the circular cone, $d_{h}$ is the average horizontal distance between $\mathrm{XR}$ and $\mathrm{CD}$, and $d_{v}$ is the vertical distance between XR and CD. Derivations for $d_{h}$ and $d_{v}$ are provided in Appendix A, whereas calculations for $\psi$ and $\phi$ are outlined in Appendix B.

Lemma 2: The expected elliptical area on 2D plane is

$$
A_{e l i p}=\pi a b=\left(\frac{\pi d_{v}^{2} \sin (\psi) \sin (2 \psi)}{2\left(\sin ^{2}(\phi)-\sin ^{2}(\psi)\right)^{\frac{3}{2}}}\right)
$$

where $\psi$ can be obtained by solving (21) and $\phi$ can be calculated from (22) given in Appendix B.

When there is no active session, i.e., $n=0$, the next session is always accepted, which implies that $Q_{1}=1$. In the rest of this section, we focus on obtaining expressions for $Q_{n+1}, n \geq 1$. To estimate $Q_{n+1}$, we formulate the following Lemma.

Lemma 3: The probability of a newly arriving $(n+1)$-th 
session to be dropped can be approximated as

$$
\begin{aligned}
Q_{n+1} & =\min \left(n\left(\frac{A_{\text {elip }}}{S_{\mathrm{R}}}\right), 1\right) \\
& =\min \left(n\left(\frac{\pi d_{v}^{2} \sin (\psi) \sin (2 \psi)}{2 S_{\mathrm{R}}\left(\sin ^{2}(\phi)-\sin ^{2}(\psi)\right)^{\frac{3}{2}}}\right), 1\right) \\
& =\min (n \gamma, 1), \text { for } n \geq 1, \\
\text { where } \gamma & =\frac{\pi d_{v}^{2} \sin (\psi) \sin (2 \psi)}{2 S_{\mathrm{R}}\left(\sin ^{2}(\phi)-\sin ^{2}(\psi)\right)^{\frac{3}{2}}} .
\end{aligned}
$$

Proof. The probability of a device emergence in the elliptical coverage area is $\frac{A_{\text {elip }}}{S_{\mathrm{R}}}$ due to our assumption on equal heights by devices of different users. Disregarding the overlap between the elliptical areas, we can assume that the total area covered by the elliptical sections in the $2 \mathrm{D}$ plane increases with $n$.

The session drop probability is directly proportional to the number of active sessions within the area of interest, which exhibits a self-limiting behavior similar to that of the population growth. While the approximation of $Q_{n+1}$ in (13) is a linear piecewise function directly proportional to $n$, due to such behavior the observed $Q_{n+1}$ is non-linear.

Theorem 1 [9]. One may further approximate $Q_{n+1}$ as

$$
\tilde{Q}_{n+1}=1-e^{-2 n \gamma} \text {. }
$$

Proof. Due to the non-linearity of the population growth, we approximate $Q_{n+1}$ with a logistic function, which has the same slope at $n=0$ as the original piecewise function. Upon further approximation, the logistic function approximation can be represented by (15). Details behind Theorem 1 can be found in [9].

With this approximation, we can further simplify the stationary distribution in (8) as follows

$$
\tilde{\pi}_{m}=\tilde{\pi}_{0} \rho^{m} \frac{e^{-\gamma m(m-1)}}{m !},
$$

where $\tilde{\pi}_{0}=\left(1+\sum_{m=1}^{\infty} \rho^{m} \frac{e^{-\gamma m(m-1)}}{m !}\right)^{-1}$ is the normalization factor.

The steady-state distribution given in (8) is characterized by a bell-shaped curve, whose maximum approximately corresponds to the expected number of sessions $E[N]$. With the approximation of the steady-state distribution and $\gamma$ given by (14), one may apply a closed-form expression for the average number of active sessions known from [9] as follows:

$$
E[N]=\frac{1}{2 \gamma} W\left(2 \gamma \rho e^{\gamma}\right),
$$

where $W(y)$ is the Lambert $\mathrm{W}$ function, a solution to $x e^{x}=y$.

Theorem 2. A lower limit on the number of co-existing sessions $N_{\min }$ if transmit power $P_{\mathrm{tx}}$ tends to infinity equals

$$
N_{\min }=\frac{1}{2 \gamma^{\prime}} W\left(2 \gamma^{\prime} \rho e^{\gamma^{\prime}}\right)
$$

where $\gamma^{\prime}=\frac{\pi d_{v}^{2} \sin (\theta) \sin (2 \theta)}{2 S_{\mathrm{R}}\left(\sin ^{2}(\phi)-\sin ^{2}(\theta)\right)^{\frac{3}{2}}}$.

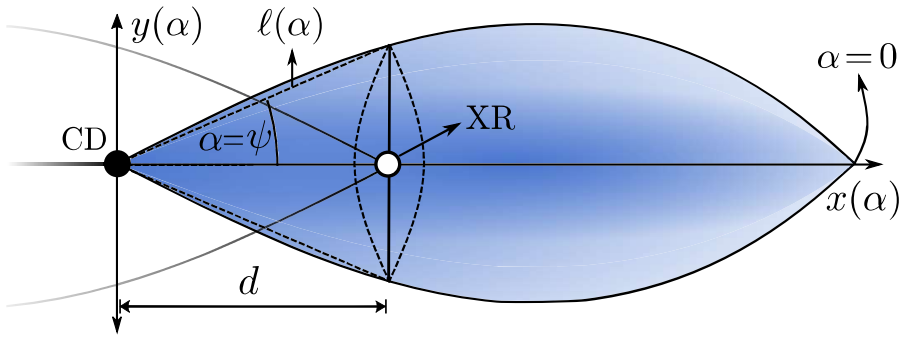

Fig. 5: Illustration of beam coverage area.

Proof. Deviation angle $\alpha$ defines the distance $\ell(\alpha)$ between the beam axis and the border of the effective beam coverage area (see Fig. 5) as

$$
\ell(\alpha)=\left(\frac{P_{\mathrm{tx}} D_{0} \rho(\alpha)}{P_{\mathrm{thr}} C}\right)^{\frac{1}{\kappa}}=R\left(1-\frac{\alpha}{\theta}\right)^{\frac{1}{\kappa}}, \text { if } \alpha \leq \theta .
$$

The $x$ - and $y$-coordinates of arbitrary points at the said border are $x(\alpha)=\ell(\alpha) \cos (\alpha)$ and $y(\alpha)=\ell(\alpha) \sin (\alpha)$, for $\alpha \leq \theta$. To obtain an expression for $N_{\min }$, which corresponds to a lower limit on the number of sessions that can exist simultaneously, we consider the limit on $x(\alpha)=\ell(\alpha) \cos (\alpha)$ w.r.t. $P_{\mathrm{tx}}$ as

$\lim _{P_{\mathrm{tx}} \rightarrow \infty} \ell(\alpha) \cos (\alpha)=\lim _{P_{\mathrm{tx}} \rightarrow \infty}\left(\frac{P_{\mathrm{tx}} D_{0}}{P_{\mathrm{thr}} C}\right)^{\frac{1}{\kappa}}\left(1-\frac{\alpha}{\theta}\right)^{\frac{1}{\kappa}} \cos (\alpha)$.

By equating the above expression to distance $d$ between the communicating devices and solving the respective equation w.r.t. $\alpha$, we derive the angle of deviation for which $E[N]$ is saturated. We conclude that $\alpha=\theta$, and by substituting $\theta$ instead of $\psi$ in (14) for $\gamma$, we may obtain $\gamma^{\prime}$.

\section{NUMERICAL RESULTS}

In this section, we report selected numerical results to illustrate the influence of the half-power beamwidth (HPBW) and transmit power on the system-level performance. Further, we also compare the cases of $2 \mathrm{D}$ and $3 \mathrm{D}$ beamforming. We model an industrial work floor environment, where workers are equipped with XR headsets and the accompanying computation devices that communicate directly using, e.g., IEEE 802.11ad/ay radio technology. All our results are verified with Monte Carlo simulations. For the analytical results, the horizontal component of the distance $d_{h}$ between the paired devices is replaced with the expected value of the horizontal distances $E\left[d_{h}\right]$, whereas in the simulations, the distance depends on the random placing of XR/CD devices around the cylinder as described in Section II. For results in this paper, we follow the FSPL model, but our proposed framework can be adapted to work with other path loss models as well. The core simulation parameters are collected in Table I.

\section{A. Impact of power and HPBW}

In Fig. 6, we detail the impact of the transmit power on the expected number of the co-existing sessions for different HPBW values. As the transmit power increases, the number of simultaneously active sessions decreases, which can be 
TABLE I: System parameters

\begin{tabular}{|l|c|c|}
\hline \hline Description & Notation & Value \\
\hline \hline Radius of area of interest & $R_{\max }$ & $20 \mathrm{~m}$ \\
\hline Carrier frequency & $f$ & $60 \mathrm{GHz}$ \\
\hline Threshold power & $P_{\mathrm{thr}}$ & $-78 \mathrm{dBm}$ \\
\hline Transmit power & $P_{\mathrm{tx}}$ & $-20,0,20 \mathrm{dBm}$ \\
\hline Propagation exponent & $\kappa$ & 2 \\
\hline Propagation constant & $C$ & $6.3 \times 10^{6}$ \\
\hline HPBW & $\theta$ & $18^{\circ}, 30^{\circ}, 52^{\circ}$ \\
\hline Arrival rate & $\lambda$ & $200 \mathrm{~s}^{-1}$ \\
\hline Height of cylinder & $h_{c}$ & $1.7 \mathrm{~m}$ \\
\hline Radius of cylinder & $r_{c}$ & $0.2 \mathrm{~m}$ \\
\hline Gap between cylinder and device & $d_{c}$ & $0.02 \mathrm{~m}$ \\
\hline Height of XR & $r_{\mathrm{XR}}$ & $1.6 \mathrm{~m}$ \\
\hline Height of CD & $r_{\mathrm{CD}}$ & $0.7 \mathrm{~m}$ \\
\hline Service rate & $\mu$ & $1 \mathrm{~s}^{-1}$ \\
\hline \hline
\end{tabular}

explained by the fact that for the lower transmit power, the radiation footprint is smaller. One can observe that after a certain point, an increase in power does not reduce the number of sessions any further, and $E[N]$ saturates at $N_{\max }$ value. For larger HPBW values, the system has a lower saturation level due to a wider spread of the radiation footprint in the horizontal plane at the same height as the devices.

Further, Fig. 7 quantifies the impact of the HPBW on the session drop probability for different levels of transmit power. For higher transmit power, as the HPBW becomes larger, the session drop probability continues to grow. The visual gap between the curves for lower transmit power $P_{\mathrm{tx}}=-20 \mathrm{dBm}$ and higher transmit power becomes wider for the increased HPBW. The probability plot for $P_{\mathrm{tx}}=-20 \mathrm{dBm}$ is nonmonotonic, and approximately at $\theta=40^{\circ}$, the session drop probability reaches its maximum. Its further decrease can be explained by the drop-shaped antenna patterns. In particular, for HPBW $\theta=40^{\circ}$, the elliptical section on the 2D plane is at its largest size, thus, resulting in a point of maximum for the session drop probability.

\section{B. Comparison of $2 D$ and $3 D$ analytical models}

We compare the results for the 2D and 3D beamforming cases. For the 2D beamforming, we follow the analysis from our previous work [9], in which all the communicating entities are considered to be located on the $2 \mathrm{D}$ horizontal plane, thus, not requiring to take into account the impact of the vertical plane. Here, Fig. 8 illustrates the dependency of the session drop probability on the transmit power for wide and narrow HPBWs in the case of 2D or 3D beamforming. One may learn that the results for the $2 \mathrm{D}$ vs. 3D cases differ considerably. For $\theta=52^{\circ}$, the session drop probability decreases by $43 \%$ and for $\theta=18^{\circ}$, the session drop probability decreases by $93 \%$. This significant drop is due to the fact that in the 3D case, the interference from neighboring devices is primarily dispersed in the vertical direction, whereas in the $2 \mathrm{D}$ case, the interference is concentrated in the $2 \mathrm{D}$ plane, thus, leading to biased results. Moreover, in the 2D case, for varied HPBW, the gap between the drop probability curves is not as wide as

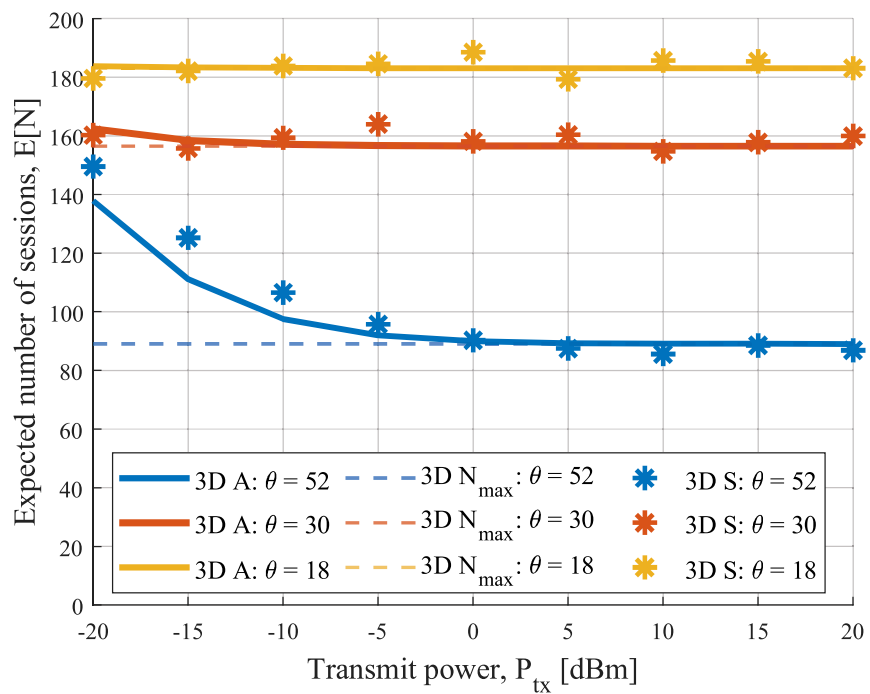

Fig. 6: Expected number of sessions $E[N]$ w.r.t. transmit power $P_{\mathrm{tx}}$ for varied HPBW $\theta$.

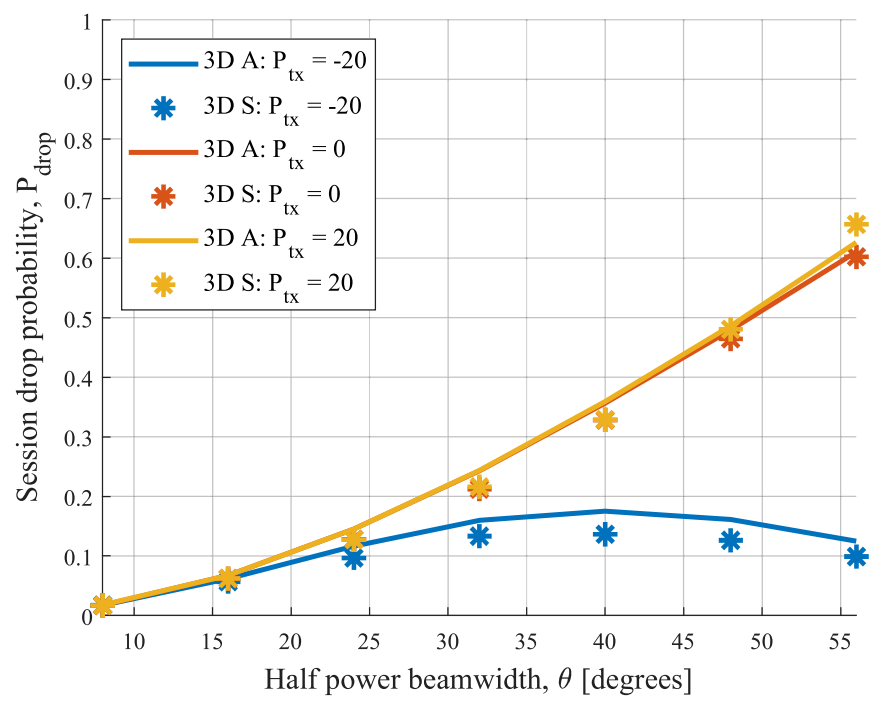

Fig. 7: Session drop probability $\mathrm{P}_{\text {drop }}$ w.r.t. HPBW $\theta$ for varied transmit power $P_{\mathrm{tx}}$.

it is for the 3D case, which highlights the importance of the HPBW optimization in the 3D setting.

In Fig. 8, we illustrate that with increased transmit power, the session drop probability eventually saturates. This is explained by the fact that after a certain level of the transmit power, the eclipse section ceases expanding, and, therefore, the drop probability in the $3 \mathrm{D}$ case stops growing. In the $2 \mathrm{D}$ case, the session drop probability continues approaching 1 . In addition, the session drop probability demonstrates two opposite trends for the $2 \mathrm{D}$ vs. $3 \mathrm{D}$ cases, if we compare wider $\theta=52^{\circ}$ and narrower $\theta=18^{\circ}$ HPBWs. In the 3D case, sessions are dropped less frequently if the HPBW is narrow, whereas in the $2 \mathrm{D}$ case, the situation is reverse. This result emphasizes the benefits of using 3D beamforming as well as the importance of studying such systems in a 3D setup. 


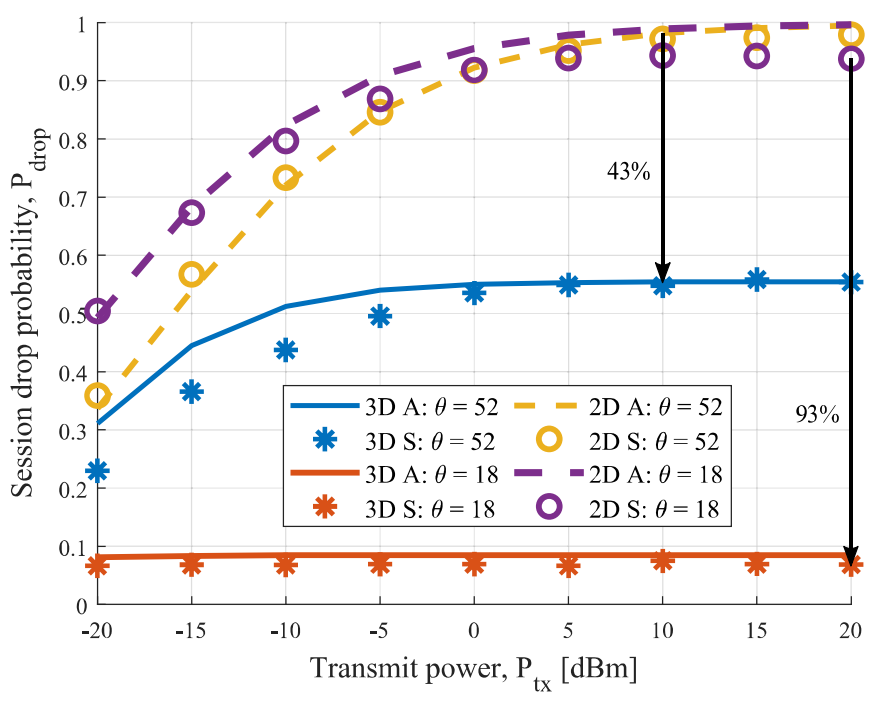

Fig. 8: Comparison of 2D and 3D beamforming w.r.t. $\mathrm{P}_{\text {drop. }}$

\section{CONCLUSION}

In this paper, we developed an analytical framework for the performance evaluation of a system of directional distributed 3D WBANs with dynamic session arrivals. Our methodology relates the system-level performance to important parameters and presents closed-form expressions, which quantify the session drop probability, the number of sessions that can run simultaneously, and the lower limit on the number of simultaneous sessions w.r.t. the transmit power.

We validated our analytical results with supportive simulations in a 3D setup, i.e., assuming 3D placement of communicating entities around a worker in an industrial work floor environment and directional beams having a volume in the 3D space. We demonstrated that wider HPBWs result in more interference at the neighboring devices, thus, reducing the number of simultaneous sessions. Studying such deployments in 2D space might yield biased results, and it is essential to assess the networks under 3D beamforming with 3D geometry considerations.

\section{APPENDIX}

\section{A. Expected horizontal distance $d_{h}$}

Here, we calculate the average distance between XR and CD. By the law of cosine,

$$
d_{h}=2\left(r_{b}+d_{b}\right) \sin \left(\frac{\varphi}{2}\right)
$$

and the expected horizontal distance can be expressed as

$$
E\left[d_{h}\right]=\frac{1}{\pi} \int_{0}^{\pi} 2\left(r_{b}+d_{b}\right) \sin \left(\frac{\varphi}{2}\right) d \varphi=\frac{4\left(r_{b}+d_{b}\right)}{\pi},
$$

whereas the vertical distance between $\mathrm{XR}$ and $\mathrm{CD}$ is readily $d_{v}=h_{\mathrm{XR}}-h_{\mathrm{CD}}$.

\section{B. Calculation of angles $\psi$ and $\phi$}

We derive an expression for $\psi$, which in Fig. 5 is the semiapex angle of the circular cone. We equate $x(\psi)$ to distance $d$ and solve it numerically w.r.t. $\psi$ as

$$
\ell(\psi) \cos (\psi)=R(1-\psi / \theta)^{\frac{1}{\kappa}} \cos (\psi)=d,
$$

which is a transcendental equation w.r.t. $\alpha$. Therefore, the sought angle of deviation $\psi$ at distance $d$ can be found numerically. The angle $\phi$ between the horizontal plane cutting the cone and the axis of the cone is then obtained as

$$
\phi=\arctan \left(\frac{d_{v}}{E\left[d_{h}\right]}\right) \text {. }
$$

\section{ACKNOWLEDGMENT}

This work is supported by EU Marie Skłodowska-Curie (project A-WEAR 813278) and the Academy of Finland (projects CROWN 317533 and IDEA-MILL 335935).

\section{REFERENCES}

[1] M. Breque, L. L. De Nul, and A. Petridis, "Industry 5.0: towards a sustainable, human-centric and resilient European industry," Luxembourg, LU: European Commission, Directorate-General for Research and Innovation, 2021.

[2] T. Masood and J. Egger, "Augmented reality in support of Industry 4.0 - implementation challenges and success factors," Robotics and Computer-Integrated Manufacturing, vol. 58, pp. 181-195, 2019.

[3] ETSI TR 126928 V16.1.0, "Extended reality (XR) in 5G (3GPP TR 26.928 version 16.1.0 Release 16)," 2021.

[4] A. Ghosh, A. Maeder, M. Baker, and D. Chandramouli, "5G evolution: A view on 5G cellular technology beyond 3GPP Release 15," IEEE Access, vol. 7, pp. 127639-127651, 2019.

[5] K. Akimoto, S. Kameda, M. Motoyoshi, and N. Suematsu, "Measurement of human body blocking at $60 \mathrm{GHz}$ for inter-network interference of mmWave WBAN," in 2017 IEEE Asia Pacific Microwave Conference (APMC), pp. 472-475, IEEE, 2017.

[6] K. Venugopal, M. C. Valenti, and R. W. Heath, "Device-to-device millimeter wave communications: Interference, coverage, rate, and finite topologies," IEEE Transactions on Wireless Communications, vol. 15, no. 9, pp. 6175-6188, 2016.

[7] G. George, K. Venugopal, A. Lozano, and R. W. Heath, "Enclosed mmWave wearable networks: Feasibility and performance," IEEE Transactions on Wireless Communications, vol. 16, no. 4, pp. 2300-2313, 2017.

[8] A. Ali, O. Galinina, and S. Andreev, "Modeling system-level dynamics of direct XR sessions over mmWave links," in 2020 IEEE 31st Annual International Symposium on Personal, Indoor and Mobile Radio Communications, pp. 1-7, 2020.

[9] A. Ali, O. Galinina, and S. Andreev, "System-level dynamics of highly directional distributed networks," IEEE Wireless Communications Letters, 2021 (in press).

[10] O. Chukhno, N. Chukhno, O. Galinina, Y. Gaidamaka, S. Andreev, and K. Samouylov, "Analysis of 3D deafness effects in highly directional mmwave communications," in 2019 IEEE Global Communications Conference (GLOBECOM), pp. 1-6, Dec 2019.

[11] O. Galinina, S. Andreev, M. Gerasimenko, Y. Koucheryavy, N. Himayat, S. p. Yeh, and S. Talwar, "Capturing spatial randomness of heterogeneous cellular/WLAN deployments with dynamic traffic," IEEE Journal on Selected Areas in Communications, vol. 32, no. 6, pp. 1083-1099, 2014.

[12] S. Andreev, O. Galinina, A. Pyattaev, K. Johnsson, and Y. Koucheryavy, "Analyzing assisted offloading of cellular user sessions onto D2D links in unlicensed bands," IEEE Journal on Selected Areas in Communications, vol. 33, no. 1, pp. 67-80, 2014

[13] H. C. Rajpoot, "Analysis of oblique frustum of a right circular cone," International Journal of Mathematics and Physical Sciences Research, 2015. 